\title{
Expanded Access Study Protocol Version
}

National Cancer Institute

\section{Source}

National Cancer Institute. Expanded Access Study Protocol Version. NCI Thesaurus.

Code $C 93485$.

An action plan of a pre-clinical or clinical study describing the procedure for obtaining an experimental drug or device for patients who are not adequately treated by existing therapy, who do not meet eligibility criteria for enrollment, or who are otherwise unable to participate in a controlled clinical study. 